\title{
Estilo de vida e indicadores antropométricos de idosas praticantes de atividade física
}

\author{
David Michel de Oliveira*, Marina Candida Lopes ${ }^{\star \star}$, Daniel dos Santos ${ }^{\star \star \star}$, Edlaine Faria de Moura Villela ${ }^{\star \star \star \star}$
}

\section{Resumo}

O objetivo deste estudo foi analisar o estilo de vida e a antropometria de idosas do projeto Geração 60+, de Sacramento, Minas Gerais. Participaram vinte idosas praticantes de atividades físicas com idade média de $68 \pm 9$ anos. Por intermédio de questionário, foram coletados dados sociodemográficos, históricos e antecedentes familiares de doenças, sintomas clínicos, estilo de vida e conhecimento sobre atividade física, tipo e frequência das atividades realizadas. As variáveis antropométricas coletadas foram o índice de massa corpórea (IMC) e relação cintura quadril (RCQ). Empregou-se estatística descritiva para análise dos dados. Observou-se que 55\% das idosas eram casadas, $85 \%$ aposentadas e $75 \%$ tinham baixo nível de escolaridade. O histórico de doenças crônicas degenerativas referiu $50 \%$ maternas e $30 \%$ paternas. As participantes apresentaram sintomas de alguma doença, $85 \%$ visitavam periodicamente o médico e usavam medicamentos. As idosas eram não fumantes e 85\% não consumiam álcool. Realizavam as principais refeições e $25 \%$ faziam dieta. Observou-se prevalência $(70 \%)$ para a prática de atividade física mais de três vezes por semana, durante 1 hora. Informaram não saber a diferença entre atividade física e exercício físico, mas reconheceram a sua importância para a saúde, $85 \%$ foram influenciadas por profissionais de saúde a realizar atividades físicas. A maioria das idosas apresentou IMC acima dos valores de referência e todas estavam com RCQ acima da normalidade. Apesar de a maioria demonstrar comportamentos preventivos e saudáveis, seus indicadores antropométricos refletiram risco à saúde. Existe uma necessidade de ações mais efetivas no projeto e de estratégias de educação em saúde, especialmente para a população de baixa escolaridade.

Palavras-chave: Antropometria. Atividade física. Envelhecimento. Hábitos. Saúde.

* Doutor em Alimentos e Nutrição pela Universidade Estadual Paulista. Mestre em Promoção de Saúde pela Universidade de Franca. Professor adjunto da Universidade Federal de Goiás. Colaborador do Programa de Pós-Graduação em Ciências Aplicadas à Saúde. Endereço para correspondência: UFG/Jataí, GO - Campus Cidade Universitária, BR 364, Km 195, 3.800, CEP 75801-615. E-mail: profdoliveira@gmail.com / davidceuclar@gmail.com

** $\quad$ Mestre em Promoção de Saúde pela Universidade de Franca. E-mail: marininhasacra@hotmail.com

*** Doutor em Alimentos e Nutrição pela Universidade Estadual Paulista. Professor do mestrado em Promoção de Saúde da Universidade de Franca, Franca, SP. E-mail: daniel.santos@unifran.edu.br

***** Doutora em Ciências pela Faculdade de Saúde Pública, Universidade de São Paulo. Mestre em Saúde Pública pela Universidade de são Paulo. Professora adjunta da Universidade Federal de Goiás, no curso de Medicina, Jataí, GO. E-mail: edlainefmv@gmail.com

$\rightarrow$ http://dx.doi.org/10.5335/rbceh.v13i2.5924

Recebido em: 02/04/2016. Aceito em: 28/06/2016. 


\section{Introdução}

Há consenso científico sobre o crescimento da população idosa no Brasil e no mundo, fato decorrente da melhora das condições de vida e do avanço da ciência no controle de doenças e suas morbimortalidades (NASRI, 2008). Conhecer o perfil sociodemográfico torna-se fundamental para fomentar elementos e informações a serem inseridas nas políticas públicas de saúde para melhorias da qualidade de vida dessa população (MENEZES; LOPES; MARUCCI, 2007).

A presença de doenças crônicas não transmissíveis em antecedentes familiares de primeiro grau pode aumentar em mais de duas vezes o risco de desenvolvimento de doenças cardiovasculares. Portanto, o levantamento pré-participação em programas de atividade física torna-se obrigatório para, assim, evitar eventos cardiovasculares (CIRUZZI et al., 1997).

Os hábitos ou o estilo de vida do indivíduo interferem diretamente na qualidade da longevidade do idoso, logo, as dimensões comportamentais, como consumo de álcool, tabagismo, controle clínico de doenças, uso de medicamentos, prática de atividade física e alimentação, são fatores que devem ser identificados a fim de propor estratégias de prevenção e intervenção na saúde senil (PENTEADO et al., 2002; INSTITUTE OF MEDICINE FOOD AND NUTRITION, 2005; DONNELLY et al., 2009; SENGER et al., 2011; ZAITUNE et al., 2012).

São necessários mais estudos referentes à prática de atividade física pela população idosa, de modo a favorecer a elaboração de programas, campanhas educativas, treinamento de profissionais de saúde e criação de espaços apropriados voltados ao estilo de vida ativo (FRANCHI, 2008).

$\mathrm{O}$ consumo inadequado de nutrientes associado à inatividade física ou ao gasto energético insuficiente podem contribuir para alterações no perfil antropométrico, aumentando o percentual de gordura abdominal, resultando em doenças cardiometabólicas (SÁNCHEZ-VIVEROS et al., 2008). Conhecer o perfil dos indicadores antropométricos em idosas participantes de projetos de atividade física seria de grande importância para avaliar sua interferência no comportamento e nos hábitos dessa população. Portanto, o objetivo deste estudo foi descrever o perfil da população-alvo, analisando o estilo de vida e a antropometria de idosas praticantes de atividades físicas.

\section{Materiais e método}

O estudo realizado caracteriza-se como descritivo transversal. Foram convidados 65 idosos de ambos os sexos que participavam do projeto institucional Geração 60+, desenvolvido pela Superintendência de Saúde e Esporte da cidade de Sacramento, Minas Gerais, Brasil. No entanto, aceitaram participar de forma voluntária vinte idosas.

O convite para participação da pesquisa foi feito pelos pesquisadores em uma reunião coletiva, esclarecendo que a participação no estudo seria espontânea e requeria o consentimento por meio da assinatura do termo de consentimento 
livre e esclarecido. A coleta de dados foi realizada no mês de outubro de 2014 , no centro de convivência de idosos da cidade de Sacramento. O projeto Geração $60+$ visa a favorecer estilo de vida saudável à população idosa por meio de atividades físicas, como caminhada, hidroginástica, ginástica e natação, realizadas com regularidade, cinco vezes por semana. A participação nas atividades físicas foi recomendada por profissionais de saúde ou foi escolha espontânea dos idosos.

Foram adotados os seguintes critérios de inclusão: idosos praticantes de atividades físicas por, no mínimo, três meses e que estivessem aptos a responder aos questionários e participar das avaliações físicas. Foram excluídos: idosos que não estivessem participando do projeto.

Para levantamento do perfil sociodemográfico e análise do estilo de vida, foi aplicado um questionário semiestruturado com múltiplas questões adaptadas à linguagem e ao nível de conhecimento da população investigada:

a) perfil sociodemográfico: idade, sexo, escolaridade e ocupação profissional;

b) histórico familiar de antecedente de doenças: doenças referidas pelas idosas e existentes em parentes de primeiro grau (pai e mãe);

c) estado clínico e uso de medicamentos: verificar sintomas de alguma doença e identificar tipos de medicamentos consumidos; d) hábitos de vida: uso de tabaco, consumo de álcool, hábitos alimentares e prática de dieta;

e) conhecimento referente a conceitos e à prática de atividade física: conceitos, importância da prática, fontes de informação e também quais modalidades são praticadas e sua regularidade.

Em relação à antropometria, para o cálculo do índice de massa corpórea (IMC) e da relação cintura quadril (RCQ), foram obtidos, inicialmente, os dados das variáveis: estatura, peso, circunferência da cintura e do quadril. A estatura foi verificada por meio de estadiômetro profissional portátil com escala de $1 \mathrm{~cm}$, marca Sanny ${ }^{\circledR}$, e o peso foi verificado por meio de balança digital, marca G-Tech ${ }^{\circledast}$.

Para a verificação do perímetro da cintura, a idosa manteve-se em pé com abdômen relaxado e os braços descontraídos ao lado do corpo, a fita foi colocada horizontalmente no ponto médio entre a borda inferior da última costela e a crista ilíaca, para verificar as medidas foi utilizada uma fita firme sobre a pele, sem compressão dos tecidos. Foi utilizada uma fita métrica inelástica da marca Sanny ${ }^{\circledR}$ com escala de $1 \mathrm{~mm}$. Para o perímetro do quadril, a fita métrica foi colocada horizontalmente em volta do quadril, na parte mais saliente dos glúteos (CALLAWAY et al., 1991).

Após a obtenção dos perímetros da cintura e do quadril, foi calculada a relação cintura quadril das idosas. Para os dados antropométricos (IMC e 
RCQ), foram utilizadas as referências da Organização Mundial de Saúde, que estabelece a seguinte classificação para o IMC: baixo peso $\left(<18,5 \mathrm{~kg} / \mathrm{m}^{2}\right)$; normal $\left(18,5-24,5 \mathrm{~kg} / \mathrm{m}^{2}\right)$; sobrepeso $(25,0-29,9$ $\left.\mathrm{kg} / \mathrm{m}^{2}\right)$; obesidade grau I $(30,0-34,9 \mathrm{~kg} /$ $\left.\mathrm{m}^{2}\right)$; obesidade grau II $\left(35,0-39,9 \mathrm{~kg} / \mathrm{m}^{2}\right)$; obesidade mórbida $\left(>40 \mathrm{~kg} / \mathrm{m}^{2}\right)$. Para 0 $\mathrm{RCQ}$, foi estabelecido que RCQ $>0,8$ $\mathrm{cm}$ indica fator de risco para doenças metabólicas e cardiovasculares (WORLD HEALTH ORGANIZATION, 2008; CABRERA et al., 2005).

O estudo adotou a estatística descritiva por meio do software Action ${ }^{\circledR}$, versão 2.9 , a fim de sistematizar os dados coletados e viabilizar a obtenção do perfil epidemiológico da população estudada. $\mathrm{O}$ projeto foi aprovado pelo Comitê de Ética em Pesquisa da Universidade de Franca sob o Protocolo nํㅡㄹ 25094814.2.0000.5495.

\section{Resultados}

Em relação ao perfil sociodemográfico, as vinte idosas analisadas no presente estudo tinham idade média de $68 \pm 9$ anos, $85 \%$ eram aposentadas, $75 \%$ apresentaram nível de escolaridade de $1^{\text {a }}$ a $4^{\mathrm{a}}$ séries e $55 \%$ eram casadas.

Quanto à amostra entrevistada, 50\% informaram algum tipo de patologia em suas progenitoras maternas $(15 \%$ diabetes, $15 \%$ doenças cardiovasculares, $10 \%$ hipertensão arterial sistêmica, 5\% câncer e 5\% obesidade), $30 \%$ relataram algum tipo de patologia no progenitor paterno (10\% hipertensão arterial sistêmica, $15 \%$ doenças cardiovasculares e $5 \%$ outros tipos de doenças). Considera- -se que as maiores prevalências de doenças foram encontradas nas mães das idosas. De acordo com a análise realizada no estudo, quando somados os dados referentes a doenças dos antecedentes familiares (pai e mãe), $80 \%$ das idosas afirmaram que o pai ou a mãe foram acometidos por algum tipo de doença crônica não transmissível.

Foi possível observar uma ou mais doenças citadas pelas entrevistadas, como disfunção tiroideana (15\%), obesidade (5\%), hipertensão arterial sistêmica (50\%), osteoporose (50\%), doenças cardiovasculares (15\%), diabetes (20\%), hipercolesterolemia (40\%), dentre outros agravos.

A grande maioria (85\%) visitava o médico periodicamente. Quanto aos sintomas de doenças silenciosas e multicausais, $15 \%$ informaram sentir falta de ar frequente em atividades da vida diária e em atividades físicas, como pedalar e caminhar, $35 \%$ sentiam dores no peito, fadiga muscular e ansiedade, $30 \%$ relataram tosse excessiva, $15 \%$ apresentaram, em algum momento, palpitações, formigamentos, tonturas, depressão ou sofreu alguma queda no último ano, e $10 \%$ queixaram-se de cefaleia ou perturbações visuais. Em relação ao uso de medicamentos, $95 \%$ utilizavam algum fármaco para tratamento de doenças, sendo a maioria para controle da hipertensão arterial (40\%) e tratamento da osteoporose (30\%).

A respeito dos hábitos e comportamentos prejudiciais à saúde, observou-se que $100 \%$ das idosas eram não fumantes. Quanto à ingestão de álcool, 85\% não 
consumiam álcool e 15\% faziam uso em pequenas doses uma vez por semana.

Hábitos alimentares e realização de refeições também foram abordados no referido estudo: 90\% tomavam café da manhã, $30 \%$ faziam colação entre o café e o almoço, $100 \%$ tinham hábito de almoçar. Desse total, $85 \%$ tomavam o lanche no período vespertino, $70 \%$ tinham o hábito de jantar e $35 \%$ realizavam a ceia antes de dormir. Foi questionado sobre a prática de dietas (controle alimentar) e seus objetivos, como emagrecimento, terapêutica ou para ganho de peso, $25 \%$ informaram fazer dieta para emagrecimento e controle de variáveis metabólicas, como glicemia e colesterol.

Quanto ao conhecimento e à prática de atividade física, $80 \%$ disseram não conhecer a diferença entre atividade física e exercício físico, entretanto, $100 \%$ responderam que a prática regular de atividade física tem importância para a saúde, e que obtiveram essas informações de profissionais ( $85 \%$ médicos, $15 \%$ educadores físicos e $5 \%$ fisioterapeutas), meios de comunicação (30\% televisão, $5 \%$ jornais e revistas) e algumas disseram ter sido persuadidas por pessoas próximas $(25 \%)$.

Em relação à prática de atividades físicas que realizavam antes de participar do projeto, $30 \%$ responderam que faziam algum tipo de atividade física regular, como caminhada, natação ou ioga, apenas essa última modalidade era monitorada por profissional. Assim, anteriormente ao projeto, $70 \%$ das idosas não praticavam ou realizavam tais atividades com regularidade.
Quanto aos tipos de atividades físicas realizadas pelas idosas no projeto, observou-se uma prevalência para ginástica $(90 \%)$ e hidroginástica $(85 \%)$. Em relação à regularidade dessas atividades, $70 \%$ realizavam mais de três vezes por semana (Tabela 1).

Tabela 1 - Realização de exercícios e atividades físicas das idosas no projeto Geração 60+, Sacramento, MG, 2013

\begin{tabular}{l|r|r}
\multicolumn{1}{c|}{ Atividade física } & \multicolumn{1}{c|}{ N } & \multicolumn{1}{c}{$\%$} \\
\hline Caminhada & 08 & 40 \\
Ginástica & 18 & 90 \\
Hidroginástica & 17 & 85 \\
Natação & 05 & 25 \\
Frequência por semana & & \\
$\quad$ 2 vezes & 05 & 25 \\
3 vezes & 01 & 5 \\
$\quad$ > 3 vezes & 14 & 40 \\
\hline
\end{tabular}

Fonte: dados da pesquisa.

Sobre os benefícios adquiridos com a prática regular de atividade física no projeto, $100 \%$ obtiveram melhora na dimensão física com melhor vigor físico, diminuição do cansaço e estresse. Do ponto de vista da dimensão psicossocial, 95\% apresentaram melhora na sensação de bem-estar e nas relações pessoais entre as participantes do projeto.

De acordo com o IMC, apenas $35 \%$ das idosas encontravam-se dentro da normalidade, enquanto $65 \%$ estavam distribuídas nas faixas de pré-obeso, obeso em grau I e sobrepeso. Quanto ao indicador antropométrico RCQ, todas as participantes apresentaram risco para doenças metabólicas e cardiovasculares (Tabela 2 ). 
Tabela 2 - Dados antropométricos dos idosos, projeto Geração 60+ da Superintendência de Saúde e Esporte, Sacramento, MG, Brasil, 2014

\begin{tabular}{c|l|r|r}
\hline \multicolumn{2}{c|}{ Dados antropométricos } & \multicolumn{1}{c|}{ N } & \multicolumn{1}{c}{$\%$} \\
\hline \multirow{3}{*}{ IMC } & Normal & 07 & 35 \\
& Sobrepeso & 09 & 45 \\
& Obeso grau I & 04 & 20 \\
RCQ & Mulheres > 0,8 & 20 & 100 \\
\hline
\end{tabular}

Fonte: dados da pesquisa.

Nota: "RCQ e IMC valores de referência: (WORLD HEALTH ORGANIZATION, 2008).

\section{Discussão}

Em relação às características demográficas do grupo de idosas pesquisado, a maioria era casada, aposentada e com baixo grau de escolaridade ( $1^{\mathrm{a}}$ a $4^{\mathrm{a}}$ séries). A predominância de mulheres na população parece ocorrer devido ao aumento da taxa de mortalidade dos homens, ao maior índice de nascimento feminino e à expectativa de vida das mulheres (MENEZES; LOPES; MARUCCI, 2007; SILVA et al., 2011).

A maior participação de mulheres em eventos socioculturais está relacionada à preocupação e ao autocuidado com a saúde feminina, quando comparadas com idosos do sexo masculino, que têm menor participação em ações coletivas por questões socioculturais (ARAÚJO; BACHION, 2004).

O baixo nível de escolaridade verificado neste estudo foi semelhante ao de outros achados. Considera-se que, possivelmente, essa baixa escolaridade dos idosos, frequentemente constatada por diferentes pesquisadores, seja um reflexo da taxa de alfabetização dos anos 1920 a 1940, quando não havia cobrança por nível escolar e o papel das mulheres na sociedade era casar, ter filhos e cuidar da casa (MENEZES; LOPES; MARUCCI, 2007; CAMARANO et al., 2005).

Em nossos achados, $30 \%$ das idosas entrevistadas faziam uso de medicamentos para osteoporose. Com o avanço da idade, é muito frequente o aparecimento dessa patologia devido ao decréscimo de massa óssea que ocorre no decorrer dos anos, sendo mais frequente em mulheres e podendo ocasionar fragilidade óssea, quedas e fraturas graves, limitando a mobilidade e aumentando a dependência motora e social (KOHRT et al., 2004). Contudo, um estilo de vida ativo pode influenciar na promoção da saúde, na melhoria da qualidade de vida e na prevenção ou redução da osteoporose (FERREIRA; DIETTRICH; PEDRO, 2015).

Outras doenças metabólicas foram observadas na população estudada, como hipertensão arterial, hipercolesterolemia e diabetes. Um estudo na população mexicana, com 7.322 indivíduos, indicou que o IMC e a obesidade abdominal estão associados a um aumento da prevalência de doenças metabólicas como diabetes tipo 2 e hipertensão arterial nos idosos daquele país (SÁNCHEZ-VIVEROS et al., 2008).

Vargas, Lara e Mello-Carpes (2014) estudaram a relação de diabetes, prática de exercício físico e atividades recreativas com a função cognitiva e a emotividade em grupos de terceira idade $(75 \%$ mulheres e $25 \%$ homens) com idade média de 72,63 $\pm 6,84$ anos. Os idosos 
diabéticos não participantes de grupos de terceira idade apresentaram menores escores cognitivos quando comparados com não diabéticos participantes desses grupos. Esse resultado confirma que um estilo de vida sedentário pode representar um fator de risco para aceleração de perdas cognitivas que acompanham o envelhecimento.

O histórico familiar de parentes de primeiro grau das idosas estudadas demonstrou prevalência de hipertensão e doença cardiovascular, tanto no pai quanto na mãe das idosas. Os dados referentes à hipertensão arterial foram semelhantes aos observados por Pavarini et al. (2008), ao entrevistarem 49 idosos.

No estudo de Barroso et al. (2008), $50 \%$ das idosas eram hipertensas, faziam o controle da doença por meio de medicamentos anti-hipertensivos e participavam de programas de atividade física, recurso considerado importante como estratégia não medicamentosa no controle da hipertensão.

$\mathrm{Na}$ população estudada, a grande maioria realizava acompanhamento médico periódico e fazia uso de medicamentos habitualmente. Os idosos constituem $50 \%$ dos multiusuários do Sistema Único de Saúde em decorrência das desadaptações e da vulnerabilidade biológica inerente ao envelhecimento, dessa forma, o alto consumo de medicamentos acompanha a tendência do envelhecimento populacional, sendo considerado comportamento habitual na clínica médica (PENTEADO et al., 2002).

Associadas às alterações decorrentes do envelhecimento, as mudanças psicos- sociais, como aposentadoria, perda de familiares e amigos, solidão e isolamento social, deixam a população idosa vulnerável e mais propensa a um estilo de vida menos saudável, com hábitos como o consumo abusivo de tabaco e álcool (SENGER et al., 2011).

O tabagismo é um importante fator de risco de morte para idosos, sendo, na atualidade, um problema de saúde pública. As idosas que participaram do presente estudo afirmaram não consumir ou fazer uso de qualquer tipo de tabaco. $\mathrm{O}$ estudo de Zaitune et al. (2012), a esse respeito, observou que a maior prevalência de fumantes é para homens sedentários na faixa etária de 60 a 69 anos.

O consumo excessivo de álcool interfere na nutrição equilibrada do idoso, quanto maior o seu consumo, menor é a qualidade nutricional, devido à disputa de sítios metabólicos entre o álcool e os substratos, o que provoca sobrecarga hepática e prejudica a absorção dos nutrientes. Entretanto, mulheres idosas têm menor prevalência de tabagismo e consumo de álcool, podendo refletir a maior longevidade dessa população em relação aos homens (MOREIRA; MARTINIANO FILHO, 2008).

O consumo moderado de vinho pode promover efeitos cardioprotetores em idosos, de outro modo, a alta ingestão de cerveja, mais de sete doses/semana, principalmente fora das refeições, está associada com a síndrome metabólica e baixo HDL-colesterol (VIEIRA, 2015).

Na população investigada, $15 \%$ afirmaram consumir álcool em pequenas doses ao menos uma vez por semana. 
Santos et al. (2014) investigaram a associação de hábitos de vida e dados sociodemográficos em idosos de Minas Gerais e identificaram que $12,3 \%$ consumiam bebida alcoólica, corroborando com o presente estudo. Para Vieira (2015), é importante investigar também o tipo de bebida, a quantidade e o momento do consumo, porque esses são fatores que podem interferir diretamente na saúde do indivíduo.

Ainda que a maioria das idosas tenha relatado o hábito de consumir as principais refeições do dia, não foi possível conferir a qualidade e a quantidade de alimentos consumidos, além disso, $15 \%$ das entrevistadas afirmaram manter algum tipo de dieta ou de controle alimentar. O consumo de dieta saudável tem sido amplamente recomendado para reduzir os fatores de risco de doenças crônicas degenerativas. $\mathrm{O}$ controle da glicemia plasmática, do perfil lipídico, da pressão arterial e a manutenção do peso corporal têm sido associados à ingestão equilibrada e à prática regular de exercícios físicos (INSTITUTE OF MEDICINE FOOD AND NUTRITION, 2005; DONNELLY et al., 2009).

A maioria das idosas do estudo praticava mais de uma modalidade de atividade física regularmente, por mais de três vezes por semana, durante 60 minutos, atendendo às recomendações mundiais para perda e manutenção de peso (DONNELLY et al., 2009). O hábito da prática regular de atividade física está inversamente associado ao surgimento de vários fatores de risco, complicações físicas e distúrbios psíqui- cos e sociais durante o envelhecimento (CHEIK et al., 2003).

A prática regular de atividades físicas promove ganhos na aptidão física, melhorando a funcionalidade nas atividades da vida diária e aumentando a tolerância ao esforço, além disso, atividades em grupo promovem impactos positivos para minimizar e prevenir a depressão e a ansiedade em indivíduos idosos (CHEIK et al., 2003).

Poucos trabalhos na literatura estudam o conhecimento dos idosos acerca de conceitos ou diretrizes sobre a prática regular de atividade física e quais os canais de comunicação sobre tais informações com essa população (FRANCHI, 2008). Acredita-se que esse conhecimento seria importante para fundamentar e aprimorar a implantação de programas/projetos educacionais e a criação de espaços apropriados para a promoção de saúde.

Nesta investigação, $80 \%$ afirmaram não saber a diferença entre os conceitos de atividade física e exercícios, isso pode estar relacionado ao baixo grau de escolaridade. No entanto, todas as idosas compreendiam a importância da prática regular de atividade física para a saúde. Todas as idosas sentiram-se motivadas a ter um estilo de vida ativo devido à sua autopercepção e à melhoria nos aspectos físico e mental, além disso, a maioria assegurou ter melhorado suas interações sociais com os participantes do projeto.

Foi realizado um estudo com o objetivo de verificar quais aspectos aumentam a adesão e a manutenção de idosos em programas de exercícios físicos, os resultados assinalaram que os principais 
motivos são: melhora da saúde $(84,2 \%)$; melhora do desempenho físico $(70,8 \%)$; estilo de vida saudável (62,5\%); redução do estresse $(60,8 \%)$; recuperação de lesões (55\%); melhora da autoimagem (50,8\%); melhora da autoestima, como forma de relaxamento $(47,5 \%)$. Ainda, $56,7 \%$ da população estudada foram motivadas pelos profissionais para fazer parte de programas de atividades físicas (FREITAS et al., 2007).

Quanto aos dados antropométricos, apenas $35 \%$ das idosas do estudo apresentaram IMC dentro da normalidade, enquanto o restante da amostra encontrava-se com sobrepeso (10\%), pré-obesas (35\%) e com obesidade grau I (20\%). Silveira, Kac e Barbosa (2009) investigaram a prevalência e os fatores associados à obesidade de 596 idosos acima de 60 anos, constatando a prevalência de obesidade (IMC $>30 \mathrm{~kg} / \mathrm{m}^{2}$ ) de $25,3 \%$ na população estudada, sendo $30,8 \%$ em mulheres. Quando foi estabelecido o ponto de corte de IMC $>27 \mathrm{~kg} / \mathrm{m}^{2}$, observou-se uma prevalência de $53,4 \%$ nessas mulheres. Dórea, Manochio-Pina e Santos (2015) investigaram o estado nutricional de idosos praticantes de atividade física e identificaram sobrepeso $(63,6 \%)$ na maioria da amostra estudada, constituída por 22 idosas. Esses estudos confirmam uma forte tendência de aumento do peso corporal associada à obesidade na população idosa feminina, associando-se a mudanças metabólicas durante o envelhecimento (CHEIK et al., 2003).

Dados referentes à RCQ indicaram que todas as participantes do estudo estavam com valores de circunferência acima dos recomendados, sugerindo um fator de risco para várias doenças metabólicas, como diabetes, dislipidemias e obesidade, podendo resultar em doenças cardiovasculares (CABRERA et al., 2005). Do ponto de vista clínico, a obesidade central é conhecida por gerar substâncias diabetogênicas e, portanto, deveria ser mais explorada do que o IMC (VAZQUEZ et al., 2007).

Os marcadores de gordura abdominal estão associados a alterações bioquímicas, como dislipidemias, proteinúria e aumento do ácido úrico, que ocasiona outras complicações. A maior ocorrência dessa alteração antropométrica em mulheres idosas é devido à diminuição da massa corporal magra e ao aumento da massa gorda (CABRERA; JACOB FILHO, 2001; PIERINE; NICOLA; OLIVEIRA, 2009; REIS FILHO et al., 2011).

$\mathrm{O}$ diabetes é uma doença crônica degenerativa que tem prevalência aumentada com o avanço da idade, é caracterizado por complicações agudas e crônicas, sendo considerado um problema de saúde pública emergente. Nos achados deste estudo, foi identificada prevalência de diabetes em $20 \%$ da população, além disso, os valores encontrados de RCQ foram acima dos desejáveis em todas as mulheres idosas, o que aumenta a incidência de síndrome metabólica, que é um fator de risco para o surgimento do diabetes e de doenças cardiovasculares (UMPIERRE et al., 2011).

Embora esses indicadores antropométricos sejam importantes, avaliações do percentual de gordura, da massa óssea e do perfil bioquímico seriam 
mais apropriadas para identificar o estado clínico e nutricional da população estudada. Outro ponto a ser destacado é o baixo número de participantes, que prejudica as conclusões sobre algumas variáveis, o que justifica, assim, os limites metodológicos do estudo.

\section{Conclusão}

Após análise dos dados encontrados para este estudo conclui-se que as idosas selecionadas do projeto da Secretaria de Saúde da cidade de Sacramento, MG, em sua maioria, eram casadas, com idade entre 66 e 70 anos e de baixo nível educacional.

Observa-se que o histórico de doenças dos antecedentes familiares das idosas pode influenciar no surgimento de doenças crônicas não transmissíveis, podendo ser considerado um critério de estratificação de risco para populações que participam de programas de atividade física.

As idosas, na maioria, tinham comportamentos considerados preventivos e saudáveis, como o hábito de não fumar, consumir baixa quantidade de bebida alcoólica, realizar visitas periódicas ao médico, consumir as principais refeições do dia e realizar atividades físicas semanais com duração adequada.

Em relação aos indicadores antropométricos, observou-se que grande parte da amostra do estudo apresentou o IMC acima dos valores de referência e todas as idosas tiveram a RCQ acima dos indicadores desejáveis, o que se reflete em riscos à saúde. Esses resultados podem estar associados ao consumo nutricional inadequado e ao gasto energético insuficiente.

Recomenda-se, a partir do presente estudo, que intervenções mais efetivas sejam realizadas, como incentivo à participação de idosos do sexo masculino em projetos de promoção de saúde, avaliação nutricional dos participantes, principalmente, sobre a quantidade e a qualidade da dieta, assim como realização de avaliação física e controle da intensidade das atividades para garantir melhor controle metabólico e um perfil antropométrico desejável. É necessário estabelecer estratégias de educação em saúde para esse segmento da sociedade, especialmente para aqueles de baixa escolaridade.

\section{Lifestyle and anthropometric indicators of elderly women engaged in physical activity}

\section{Abstract}

The aim of this study was to analyze the lifestyle and the anthropometry of elderly project Generation 60+ Sacramento-MG. Participated 20 elderly women engaged in physical activity with a mean age of $68 \pm 9$ years. Through questionnaire were collected demographic data, history, and family history of diseases, clinical symptoms, lifestyle and knowledge about physical activity, type and frequency of activities. On the collected anthropometric variables were body mass index (BMI) and Waist Hip Ratio (WHR). The descriptive statistics for data analysis. It was observed that $55 \%$ of older were married, $85 \%$ retired and $75 \%$ had low level of

RBCEH, Passo Fundo, v. 13, n. 2, p. 171-183, maio/ago. 2016 
education. The older history of chronic degenerative were $50 \%$ mothers diseases and $30 \%$ fathers diseases. The participants showed symptoms of an illness and $85 \%$ regularly visited the doctor and used drugs. Elderly women were non-smokers and $85 \%$ did not consume alcohol. They performed the main meals and $25 \%$ were diet. Observed prevalence $(70 \%)$ for the practice of physical activity more than three times weekly for 1 hour. Reported not knowing the difference between physical activity and exercise, but acknowledged their importance for health, $85 \%$ were influenced by health professionals to perform physical activities. Most older had BMI above the reference values and all were with WHR above normal. While most preventive and demonstrate healthy behaviors, their anthropometric indicators reflected health risk. There is a need for more effective action on the project and health education strategies especially for people with low education.

Keywords: Aging. Anthropometry. Habits. Health. Physical activity.

\section{Agradecimentos}

Os autores agradecem às participantes voluntárias entrevistadas.

\section{Referências}

ARAÚJO, L. A. O.; BACHION, M. M. Programa Saúde da Família: perfil de idosos assistidos por uma equipe. Revista Brasi- leira de Enfermagem, Brasília (DF), v. 57, n. 5, p. 586-590, out. 2004.

BARROSO, W. K. S. et al. Influência da atividade física programada na pressão arterial de idosos hipertensos sob tratamento não farmacológico. Revista da Associação Médica Brasileira, São Paulo (SP), v. 54, n. 4, p. 328333, jul./ago. 2008.

CABRERA, M. A. S.; JACOB-FILHO, W. Obesidade em idosos: prevalência, distribuição e associação com hábitos e co-morbidades. Arquivos Brasileiros Endocrinologia e Metabologia, São Paulo (SP), v. 45, n. 5, p. 494-501, out. 2001.

CABRERA, M. A. S. et al. Relação do índice de massa corporal, da relação cintura-quadril e da circunferência abdominal com a mortalidade em mulheres idosas: seguimento de 5 anos. Cadernos de Saúde Pública, Rio de Janeiro (RJ), v. 21, n. 3, p. 767-775, 2005.

CALLAWAY, C. W. et al. Circumferences. In: LOHMAN, T. G.; ROCHE, A. F.; MARTORELL, R. Anthropometric standardization reference manual. Champaign: Human Kinetics Books, 1991. p. 44-45.

CAMARANO, A. A. et al. Idosos brasileiros: indicadores de condições de vida e de acompanhamento de políticas. Brasília: Presidência da República; Subsecretaria de Direitos Humanos, 2005.

CHEIK, C. et al. Efeitos do exercício físico e da atividade física na depressão e ansiedade em indivíduos idosos. Revista Brasileira de Ciência e Movimento, Brasília (DF), v. 11, n. 3, p. 41-47, jul./set. 2003.

CIRUZZI, M. et al. Frequency of family history of acute myocardial infarction in patients with acute myocardial infarction. Argentine FRICAS Factores de Riesgo Coronario en America del Sur Investigators. The American Journal of Cardiology, New York (NY), v. 80, n. 2, p. 122-127, Jul. 1997.

DONNELLY, J. E. et al. Position stand: appropriate physical activity intervention strategies for weight loss and prevention 
of weight regain for adults. Medicine and Science in Sports and Exercise, Indianapolis, v. 41, n. 2, p. 459-471, Feb. 2009.

DÓREA, G. S.; MANOCHIO-PINA, M. G.; SANTOS, D. Aspectos nutricionais de idosos praticantes de atividade física. Demetra: Alimentação, Nutrição e Saúde, Rio de Janeiro (RJ), v. 10, n. 5, p. 347-360, 2015. Disponível em: <http://www.e-publicacoes.uerj.br/index. $\mathrm{php/demetra/article/view/14793/13278 \# .}$ V3CHk-srLIU>. Acesso em: 26 jun. 2016.

FERREIRA, J. F.; DIETTRICH, S. H. C.; PE$\mathrm{DRO}, \mathrm{D}$. A. Influência da prática de atividade física sobre a qualidade de vida de usuários do SUS. Saúde Debate, Rio de Janeiro (RJ), v. 39, n. 106, p. 792-801, jul./set. 2015.

FRANCHI, K. M. B. Estudo comparativo do conhecimento e prática de atividade física de idosos diabéticos tipo 2 e não diabéticos. $R e$ vista Brasileira de Geriatria e Gerontologia, Rio de Janeiro (RJ), v. 11, n. 3, p. 327-339, 2008.

FREITAS, C. M. S. M. et al. Aspectos motivacionais que influenciam a adesão e manutenção de idosos a programas de exercícios físicos. Revista Brasileira de Cineantropometria e Desempenho Humano, Florianópolis (SC), v. 9, n. 1, p. 92-100, mar. 2007.

INSTITUTE OF MEDICINE FOOD AND NUTRITION. Dietary reference intakes for energy, carbohydrate, fiber, fat, fatty acids, cholesterol, protein, and amino acids. Washington, DC: National Academy Press, 2005.

KOHRT, W. M. et al. Position stand: physical activity and bone health. Medicine and Science in Sports and Exercise, Indianapolis, v. 36, n. 11, p. 1985-1996, Nov. 2004.

MENEZES, T. N.; LOPES, F. J. M.; MARUCCI, M. F. N. Estudo domiciliar da população idosa de Fortaleza/CE: aspectos metodológicos e características sociodemográficas. Revista Brasileira de Epidemiologia, São Paulo (SP), v. 10, n. 2, p. 168-177, 2007.
MOREIRA, P. F. P.; MARTINIANO-FILHO, F. Aspectos nutricionais e o abuso do álcool em idosos. Envelhecimento e Saúde, São Paulo (SP), v. 14, n. 1, p. 23-26, 2008.

NASRI, F. O envelhecimento populacional no Brasil. Einstein, São Paulo (SP), v. 6, supl. 1, p. 4-6, 2008.

PAVARINI, S. C. I. et al. Genograma: avaliando a estrutura familiar de idosos de uma unidade de saúde da família. Revista Eletrônica de Enfermagem, Goiânia (GO), v. 10, n. 1, p. 39-50, 2008.

PENTEADO, P. T. P. S. et al. O uso de medicamentos por idosos. Visão Acadêmica, Curitiba (PR), v. 3, n. 1, p. 35-42, 2002.

PIERINE, D. T.; NICOLA, M.; OLIVEIRA, E. P. Sarcopenia: alterações metabólicas e consequências no envelhecimento. Revista Brasileira de Ciência e Movimento, Brasília (DF), v. 17, n. 3, p. 96-103, 2009.

REIS-FILHO, A. D. R. et al. Associação entre variáveis antropométricas, perfil glicêmico e lipídico em mulheres idosas. Revista Brasileira de Geriatria e Gerontologia, Rio de Janeiro (RJ), v. 14, n. 4, p. 675-786, 2011.

SÁNCHEZ-VIVEROS, S. et al. Association between diabetes mellitus and hypertension with anthropometric indicators in older adults: results of the Mexican Health Survey, 2000. The Journal of Nutrition Health \& Aging, New York (NY), v. 12, n. 5, p. 327333, 2008.

SANTOS, A. S. et al. Atividade física, álcool e tabaco entre idosos. Revista Família, Ciclos de Vida e Saúde no Contexto Social, Uberaba (MG), v. 2, n. 1, p. 6-13, 2014.

SENGER, A. E. et al. Alcoolismo e tabagismo em idosos: relação com ingestão alimentar e aspectos socioeconômicos. Revista Brasileira de Geriatria e Gerontologia, Rio de Janeiro (RJ), v. 14, n. 4, p. 713-719, 2011. 
SILVA, H. O. et al. Perfil epidemiológico de idosos frequentadores de grupos de convivência no município de Iguatu, Ceará. Revista Brasileira de Geriatria e Gerontologia, Rio de Janeiro (RJ), v. 14, n. 1, p. 123-133, 2011.

SILVEIRA, E. A.; KAC, G.; BARBOSA, L. $\mathrm{S}$. Prevalência e fatores associados à obesidade em idosos residentes em Pelotas, Rio Grande do Sul, Brasil: classificação da obesidade segundo dois pontos de corte do índice de massa corporal. Cadernos de Saúde Pública, Rio de Janeiro (RJ), v. 25, n. 7, p. 1.569-1.577, jul. 2009. Disponível em: <http:// www.scielosp.org/scielo.php?script $=$ sci $_{-}$ arttext\&pid=S0102-311X2009000700015\&l ng=en\&nrm=iso $>$. Acesso em: 26 jun. 2016.

UMPIERRE, D. et al. Physical activity advice only or structured exercise training and association with HbA1c levels in type 2 diabetes: a systematic review and meta-analysis. Journal of the American Medical Association, Chicago, v. 305, n. 17, p. 1790-1799, 2011.

VARGAS, L. S.; LARA, M. V. S.; MELLO-CARPES, P. B. Influência da diabetes e a prática de exercício físico e atividades cognitivas e recreativas sobre a função cognitiva e emotividade em grupos de terceira idade. Revista Brasileira de Geriatria e Gerontologia, Rio de Janeiro (RJ), v. 17, n. 4, p. 867878, 2014.

VAZQUEZ, G. et al. Comparison of body mass index, waist circumference, and waist/hip ratio in predicting incident diabetes: a meta-analysis. Epidemiologic Reviews, Baltimore, v. 19, n. 1, p. 115-128, 2007.

VIEIRA, B. A. Associação entre o consumo de álcool e síndrome metabólica: análise transversal da linha de base do Elsa-Brasil. 2015. Tese (Mestrado em Epidemiologia) Programa de Pós-Graduação em Epidemiologia, Universidade Federal do Rio Grande do Sul, Porto Alegre, 2015.

WORLD HEALTH ORGANIZATION. Waist circumference and waist-hip ratio: report of a WHO expert consultation. Geneva: WHO, 2008. Disponível em: <http://apps.who.int/iris/ bitstream/10665/44583/1/9789241501491_ eng.pdf $>$. Acesso em: 26 jun. 2016.

ZAITUNE, M. P. A. et al. Fatores associados ao tabagismo em idosos: Inquérito de Saúde no Estado de São Paulo ISA-SP. Cadernos de Saúde Pública, Rio de Janeiro (RJ), v. 28, n. 3, p. 583-596, 2012. 